\title{
Hamiltonian Submanifolds of Regular Polytopes
}

\author{
Felix Effenberger • Wolfgang Kühnel
}

Received: 18 June 2008 / Revised: 17 February 2009 / Accepted: 23 February 2009 /

Published online: 31 March 2009

(C) Springer Science+Business Media, LLC 2009

\begin{abstract}
We investigate polyhedral $2 k$-manifolds as subcomplexes of the boundary complex of a regular polytope. We call such a subcomplex $k$-Hamiltonian if it contains the full $k$-skeleton of the polytope. Since the case of the cube is well known and since the case of a simplex was also previously studied (these are so-called superneighborly triangulations), we focus on the case of the cross polytope and the sporadic regular 4-polytopes. By our results the existence of 1-Hamiltonian surfaces is now decided for all regular polytopes. Furthermore we investigate 2-Hamiltonian 4manifolds in the $d$-dimensional cross polytope. These are the "regular cases" satisfying equality in Sparla's inequality. In particular, we present a new example with 16 vertices which is highly symmetric with an automorphism group of order 128 . Topologically it is homeomorphic to a connected sum of seven copies of $S^{2} \times S^{2}$. By this example all regular cases of $n$ vertices with $n<20$ or, equivalently, all cases of regular $d$-polytopes with $d \leq 9$ are now decided.
\end{abstract}

Keywords Hamiltonian subcomplex · Centrally-symmetric $\cdot$ Tight $\cdot$ PL-taut Intersection form $\cdot$ Pinched surface $\cdot$ Sphere products

\section{Introduction and Results}

The idea of a Hamiltonian circuit in a graph can be generalized to higher-dimensional complexes as follows: A subcomplex $A$ of a polyhedral complex $K$ is called $k$ Hamiltonian ${ }^{1}$ if $A$ contains the full $k$-dimensional skeleton of $K$. It seems that this

\footnotetext{
${ }^{1}$ Not to be confused with the notion of a $k$-Hamiltonian graph [18].

F. Effenberger · W. Kühnel (凶)

Institut für Geometrie und Topologie, Universität Stuttgart, 70550 Stuttgart, Germany

e-mail:kuehnel@mathematik.uni-stuttgart.de

F. Effenberger

e-mail: effenberger@mathematik.uni-stuttgart.de
} 
concept was first developed by Schulz [38, 39]. A Hamiltonian circuit then becomes a special case of a 0 -Hamiltonian subcomplex of a one-dimensional graph or of a higher-dimensional complex [12]. If $K$ is the boundary complex of a convex polytope, then this concept becomes particularly interesting and quite geometrical [20, Chap. 3]. Altshuler [1] investigated 1-Hamiltonian closed surfaces in special polytopes. A triangulated surface with a complete edge graph $K_{n}$ can be regarded as a 1-Hamiltonian subcomplex of the simplex with $n$ vertices. These are the so-called regular cases in Heawood's Map Color Theorem [36], [20, 2C], and people talk about the uniquely determined genus of the complete graph $K_{n}$ which is (in the orientable regular cases $n \equiv 0,3,4,7(12), n \geq 4)$

$$
g=\frac{1}{6}\left(\begin{array}{c}
n-3 \\
2
\end{array}\right)
$$

Moreover, the induced piecewise linear embedding of the surface into Euclidean ( $n-$ 1)-space then has the two-piece property, and it is tight [20, 2D].

Centrally-symmetric analogues can be regarded as 1-Hamiltonian subcomplexes of cross polytopes or other centrally symmetric polytopes, see [21]. Similarly we have the genus of the d-dimensional cross polytope [17] which is (in the orientable regular cases $d \equiv 0,1$ (3), $d \geq 3$ )

$$
g=\frac{1}{3}(d-1)(d-3) .
$$

There are famous examples of quadrangulations of surfaces originally due to H.S.M. Coxeter which can be regarded as 1-Hamiltonian subcomplexes of higherdimensional cubes [20, 25, 2.12]. Accordingly one talks about the genus of the $d$-cube (or rather its edge graph) which is (in the orientable case)

$$
g=2^{d-3}(d-4)+1
$$

see $[5,35]$. However, in general the genus of a 1-Hamiltonian surface in a convex $d$-polytope is not uniquely determined, as pointed out in [38, 39]. This uniqueness seems to hold especially for regular polytopes, where the regularity allows a computation of the genus by a simple counting argument.

In the cubical case there are higher-dimensional generalizations by Danzer's construction of a power complex $2^{K}$ for a given simplicial complex $K$. In particular there are many examples of $k$-Hamiltonian $2 k$-manifolds as subcomplexes of higherdimensional cubes, see [25]. For obtaining them, one just has to start with a neighborly simplicial $(2 k-1)$-sphere $K$. A large number of the associated complexes $2^{K}$ are topologically connected sums of copies of $S^{k} \times S^{k}$. This seems to be the standard case.

Concerning triangulations of manifolds, a $d$-dimensional simplicial complex is called a combinatorial $d$-manifold if the union of its simplices is homeomorphic to a $d$-manifold and if the link of each $k$-simplex is a combinatorial $(d-k-1)$-sphere. In what follows all triangulations of manifolds are assumed to be combinatorial. There exist triangulations of manifolds which are not combinatorial; for an example based on the Edwards sphere, see [6]. 
With respect to the simplex as the ambient polytope a $k$-Hamiltonian subcomplex is also called a $(k+1)$-neighborly triangulation since any $k+1$ vertices are common neighbors in a $k$-dimensional simplex. The crucial case is the case of $(k+1)$ neighborly triangulations of $2 k$-manifolds. This case was studied by the second author in [20]. One could call this the case of super-neighborly triangulations in analogy with neighborly polytopes: The boundary complex of a $(2 k+1)$-polytope can be at most $k$-neighborly unless it is a simplex. However, combinatorial $2 k$-manifolds can go beyond $k$-neighborliness, depending on the topology. Except for the trivial case of the boundary of a simplex itself, there are only a finite number of known examples of super-neighborly triangulations, reviewed in [27]. They are necessarily tight [20, Chap. 4], compare Sect. 5 below. The most significant ones are the unique 9-vertex triangulation of the complex projective plane [23, 24], a 16-vertex triangulation of a $K 3$ surface [9], and several 15-vertex triangulations of an 8-manifold "like the quaternionic projective plane" [8]. There is also an asymmetric 13-vertex triangulation of $S^{3} \times S^{3}$, but most of the examples are highly symmetric. For any $n$-vertex triangulation of a $2 k$-manifold $M$, the generalized Heawood inequality

$$
\left(\begin{array}{c}
n-k-2 \\
k+1
\end{array}\right) \geq\left(\begin{array}{c}
2 k+1 \\
k+1
\end{array}\right)(-1)^{k}(\chi(M)-2)
$$

was conjectured in [19, 20] and later almost completely proved by Novik in [32] and [34]. The equality holds precisely in the case of super-neighborly triangulations. These are $k$-Hamiltonian in the $(n-1)$-dimensional simplex. In the case of 4-manifolds (i.e., $k=2$ ) an elementary proof was already contained in [20, 4B].

In the case of 2-Hamiltonian subcomplexes of cross polytopes, the first nontrivial example was constructed by Sparla as a centrally-symmetric 12-vertex triangulation of $S^{2} \times S^{2}$ as a subcomplex of the boundary of the 6-dimensional cross polytope $[29,41]$. Sparla also proved the following analogous Heawood inequality for the case of 2-Hamiltonian 4-manifolds in centrally symmetric $d$-polytopes:

$$
\left(\begin{array}{c}
\frac{1}{2}(d-1) \\
3
\end{array}\right) \leq 10(\chi(M)-2)
$$

and the opposite inequality for centrally-symmetric triangulations with $n=2 d$ vertices.

Higher-dimensional examples were found by Lutz [30]: There are centrallysymmetric 16-vertex triangulations of $S^{3} \times S^{3}$ and 20-vertex triangulations of $S^{4} \times S^{4}$. The 2-dimensional example in this series is the well-known unique centrallysymmetric 8 -vertex torus [21,3.1]. All these are tightly embedded into the ambient Euclidean space [27]. The generalized Heawood inequality for centrally symmetric $2 d$-vertex triangulations of $2 k$-manifolds

$$
4^{k+1}\left(\begin{array}{c}
\frac{1}{2}(d-1) \\
k+1
\end{array}\right) \geq\left(\begin{array}{c}
2 k+1 \\
k+1
\end{array}\right)(-1)^{k}(\chi(M)-2)
$$

was conjectured by Sparla in [42] and later almost completely proved by Novik in [33]. 
In the present paper we show that Sparla's inequality for 2-Hamiltonian 4manifolds in the skeletons of $d$-dimensional cross polytopes is sharp for $d \leq 9$. More precisely, we show that each of the regular cases (that is, the cases of equality) for $d \leq 9$ really occurs. Since the cases $d=7$ and $d=9$ are not regular, the crucial point is the existence of an example for $d=8$ and, necessarily, $\chi=16$. In addition we examine the case of 1-Hamiltonian surfaces in the three sporadic regular 4-polytopes, see Sect. 2. It seems that so far no decision about existence or nonexistence could be made, compare [40].

\section{Main Theorem}

1. All cases of 1-Hamiltonian surfaces in the regular polytopes are decided. In particular there are no 1-Hamiltonian surfaces in the 24-cell, 120-cell, or 600-cell.

2. All cases of 2-Hamiltonian 4-manifolds in the regular d-polytopes are decided up to dimension $d=9$. In particular, there is a new example of a 2-Hamiltonian 4-manifold in the boundary complex of the 8-dimensional cross polytope.

This follows from certain known results and a combination of Propositions 1, 2, and 3 and Theorem 2 below.

The regular cases of 1-Hamiltonian surfaces are the following, and each case occurs:

$$
\begin{array}{lll}
d \text {-simplex: } & d \equiv 0,2(3) & {[36]} \\
d \text {-cube: } & \text { any } d \geq 3 & {[5,35]} \\
d \text {-octahedron: } & d \equiv 0,1(3) & {[17] .}
\end{array}
$$

The regular cases of 2-Hamiltonian 4-manifolds for $d \leq 9$ are the following:

$$
\begin{array}{lll}
d \text {-simplex: } & d=5,8,9 & {[24]} \\
d \text {-cube: } & d=5,6,7,8,9 & {[25]} \\
d \text {-octahedron: } & d=5,6,8 & \text { Theorem } 2 .
\end{array}
$$

Here each of these cases occurs except for the case of the 9-simplex [24]. Furthermore 2-Hamiltonian 4-manifolds in the $d$-cube are known to exist for any $d \geq 5$ [25]. In the case of the $d$-simplex the next regular case $d=13$ is undecided, and the case $d=15$ occurs [9]. The next regular case of a $d$-octahedron is the case $d=10$, see Remark 2 below.

\section{Hamiltonian Surfaces in the 24-Cell, 120-Cell, 600-Cell}

There are Hamiltonian cycles in each of the Platonic solids. The numbers of distinct Hamiltonian cycles (modulo symmetries of the solid itself) are 1, 1, 2, 1, 17 for the cases of the tetrahedron, cube, octahedron, dodecahedron, icosahedron, see [15, pp. $277 \mathrm{ff}$.]. A 1-Hamiltonian surface in the boundary complex of a Platonic solid must coincide with the boundary itself and is, therefore, not really interesting. 
Table 1 Automorphism groups of the Hamiltonian pinched surfaces in the 24-cell

\begin{tabular}{|c|c|c|c|}
\hline Type & Group & Order & Generators \\
\hline 1 & $C_{4} \times C_{2}$ & 8 & $\begin{array}{l}(1121618)(217237)(3132021)(422115)(619)(8241410), \\
(13)(48)(510)(915)(1114)(1213)(1620)(1821)(2224)\end{array}$ \\
\hline 2 & $D_{8}$ & 8 & $\begin{array}{l}(116)(217)(322)(520)(69)(723)(812)(1024)(1418)(1519), \\
(23)(46)(57)(911)(1214)(1315)(1720)(1921)(2223)\end{array}$ \\
\hline 3 & $C_{2} \times C_{2}$ & 4 & $\begin{array}{l}(124)(213)(315)(417)(519)(620)(721)(922)(1123), \\
(25)(37)(49)(611)(818)(1319)(1521)(1722)(2023)\end{array}$ \\
\hline 4 & $\left(\left(\left(C_{4} \times C_{2}\right): C_{2}\right): C_{2}\right): C_{2}$ & 64 & $\begin{array}{l}(181012)(31354)(615199)(717)(11202122)(14241816), \\
(23)(46)(57)(911)(1214)(1315)(1720)(1921)(2223)\end{array}$ \\
\hline 5 & $S_{3}$ & 6 & $\begin{array}{l}(13)(48)(510)(915)(1114)(1213)(1620)(1821)(2224), \\
(12215)(21213)(3924)(4178)(51910)(61620)(71821)(112314)\end{array}$ \\
\hline 6 & $C_{2} \times D_{8}$ & 16 & $\begin{array}{l}(111)(223)(314)(416)(518)(820)(1021)(1222)(1324), \\
(15)(312)(410)(619)(79)(813)(1118)(1422)(1517)(1621)(2024), \\
(13)(48)(510)(915)(1114)(1213)(1620)(1821)(2224)\end{array}$ \\
\hline
\end{tabular}

Hamiltonian cycles in the regular 4-polytopes are known to exist. However, it seems that 1-Hamiltonian surfaces in the 2-skeleton of any of the three sporadic regular 4-polytopes have not yet been systematically investigated. A partial attempt can be found in [40].

\subsection{The 24-Cell}

The boundary complex of the 24-cell $\{3,4,3\}$ consists of 24 vertices, 96 edges, 96 triangles, and 24 octahedra. Any 1-Hamiltonian surface (or pinched surface) must have 24 vertices, 96 edges, and, consequently, 64 triangles; hence it has Euler characteristic $\chi=-8$. Every edge in the polytope is in three triangles. Hence we must omit exactly one of them in each case for getting a surface where every edge is in two triangles. Since the vertex figure in the polytope is a cube, each vertex figure in the surface is a Hamiltonian circuit of length 8 in the edge graph of a cube. It is well known that this circuit is uniquely determined up to symmetries of the cube. Starting with one such vertex figure, there are four missing edges in the cube, which, therefore, must be in the uniquely determined other triangles of the 24-cell. In this way, one can inductively construct an example or, alternatively, verify the nonexistence. If singular vertices are allowed, then the only possibility is a link which consists of two circuits of length four each. This leads to the following proposition.

Proposition 1 There is no 1-Hamiltonian surface in the 2-skeleton of the 24-cell. However, there are six combinatorial types of strongly connected 1-Hamiltonian pinched surfaces with a number of pinch points ranging between 4 and 10 and with the genus ranging between $g=3$ and $g=0$. The case of the highest genus is a sur- 
Table 2 Generating orbits of the 6 types of Hamiltonian pinched surfaces in the 24-cell

\begin{tabular}{llll}
\hline Type & \# p.pts. & $g$ & Orbits \\
\hline 1 & 10 & 0 & $\langle 123\rangle_{4},\langle 124\rangle_{8},\langle 136\rangle_{4},\langle 149\rangle_{8},\langle 157\rangle_{8},\langle 159\rangle_{8},\langle 1611\rangle_{8},\langle 1711\rangle_{8},\langle 2510\rangle_{4},\langle 468\rangle_{4}$ \\
2 & 10 & 0 & $\langle 123\rangle_{4},\langle 124\rangle_{8},\langle 149\rangle_{4},\langle 238\rangle_{4},\langle 2412\rangle_{8},\langle 2510\rangle_{4},\langle 2512\rangle_{4},\langle 2813\rangle_{8}$, \\
& & $\langle 21013\rangle_{8},\langle 468\rangle_{4},\langle 81315\rangle_{4},\langle 101319\rangle_{4}$
\end{tabular}

face of genus three with four pinch points. The link of each of the pinch points in any of these types is the union of two circuits of length four.

The six types and their automorphism groups are listed in Tables 1 and 2, where the labeling of the vertices of the 24-cell coincides with the standard one in polymake [13].

Type 1 is a pinched sphere which is based on a subdivision of the boundary of the rhombidodecahedron, see Fig. 1 (left). Type 4 is just a $(4 \times 4)$-grid square torus where each square is subdivided by an extra vertex, see Fig. 1 (right). These 16 extra vertices are identified in pairs, leading to the 8 pinch points.

Because -8 equals the Euler characteristic of the original (connected) surface minus the number of pinch points, it is clear that we can have at most 10 pinch points unless the surface splits into several components. We present here in more detail Type 6 as a surface of genus three with four pinch points, see Fig. 3 (produced with JavaView). Its combinatorial type is given by the following list of 64 triangles:

$\begin{array}{llllllll}\langle 123\rangle, & \langle 124\rangle, & \langle 136\rangle, & \langle 149\rangle, & \langle 157\rangle, & \langle 159\rangle, & \langle 1611\rangle, & \langle 1711\rangle, \\ \langle 238\rangle, & \langle 248\rangle, & \langle 2510\rangle, & \langle 2512\rangle, & \langle 21013\rangle, & \langle 21213\rangle, & \langle 3614\rangle, & \langle 3710\rangle, \\ \langle 3714\rangle, & \langle 3815\rangle, & \langle 31015\rangle, & \langle 468\rangle, & \langle 4616\rangle, & \langle 4912\rangle, & \langle 41217\rangle, & \langle 41617\rangle, \\ \langle 5710\rangle, & \langle 5918\rangle, & \langle 51219\rangle, & \langle 51819\rangle, & \langle 6820\rangle, & \langle 61114\rangle, & \langle 61620\rangle, & \langle 71118\rangle, \\ \langle 71421\rangle, & \langle 71821\rangle, & \langle 81315\rangle, & \langle 81317\rangle, & \langle 81720\rangle, & \langle 91116\rangle, & \langle 91118\rangle, & \langle 91222\rangle, \\ \langle 91622\rangle, & \langle 101319\rangle, & \langle 101521\rangle, & \langle 101921\rangle, & \langle 111423\rangle, & \langle 111623\rangle, & \langle 121317\rangle, & \langle 121922\rangle, \\ \langle 131524\rangle, & \langle 131924\rangle, & \langle 141520\rangle, & \langle 141521\rangle, & \langle 142023\rangle, & \langle 152024\rangle, & \langle 161722\rangle, & \langle 162023\rangle, \\ \langle 172024\rangle, & \langle 172224\rangle, & \langle 181922\rangle, & \langle 182123\rangle, & \langle 182223\rangle, & \langle 192124\rangle, & \langle 212324\rangle, & \langle 222324\rangle .\end{array}$

The pinch points are the vertices 2, 6, 19, 23 with the following links: 

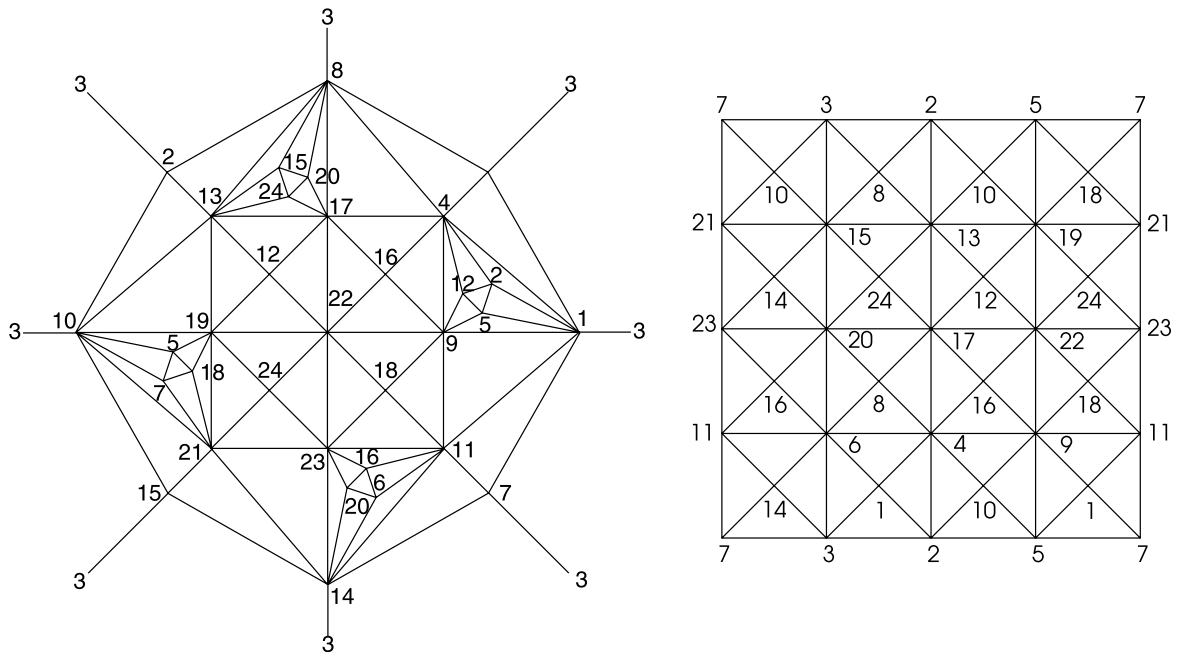

Fig. 1 Type 1 (left) and Type 4 (right) of Hamiltonian pinched surfaces in the 24-cell

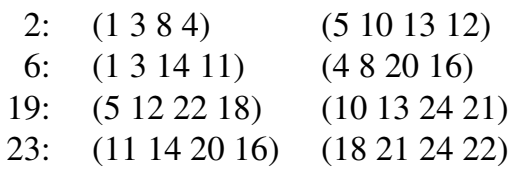

The four vertices 7, 9, 15, 17 are not joined to one another and not to any of the pinch points either. Therefore the eight vertex stars of 7, 9, 15, 17, 2, 6, 19, 23 cover the 64 triangles of the surface entirely and simply, compare Fig. 2 where the combinatorial type is sketched. In this drawing all vertices are 8-valent except for the four pinch points in the two "ladders" on the right-hand side which have to be identified in pairs.

The combinatorial automorphism group of order 16 is generated by

$$
\begin{aligned}
& Z=(111)(223)(314)(416)(518)(820)(1021)(1222)(1324), \\
& A=(15)(312)(410)(619)(79)(813)(1118)(1422)(1517)(1621)(2024), \\
& B=(13)(48)(510)(915)(1114)(1213)(1620)(1821)(2224) .
\end{aligned}
$$

The elements $A$ and $B$ generate the dihedral group $D_{8}$ of order 8 , whereas $Z$ commutes with $A$ and $B$. Therefore the group is isomorphic with $D_{8} \times C_{2}$.

\subsection{The 120-Cell and the 600-Cell}

The 600 -cell has the $f$-vector $(120,720,1200,600)$, and by duality the 120 -cell has the $f$-vector $(600,1200,720,120)$. Any 1 -Hamiltonian surface in the 600 -cell must have 120 vertices, 720 edges, and, consequently, 480 triangles (namely, two out of five), so it has Euler characteristic $\chi=-120$ and genus $g=61$. We obtain the same genus in the 120 -cell by counting 600 vertices, 1200 edges, and 480 pentagons 

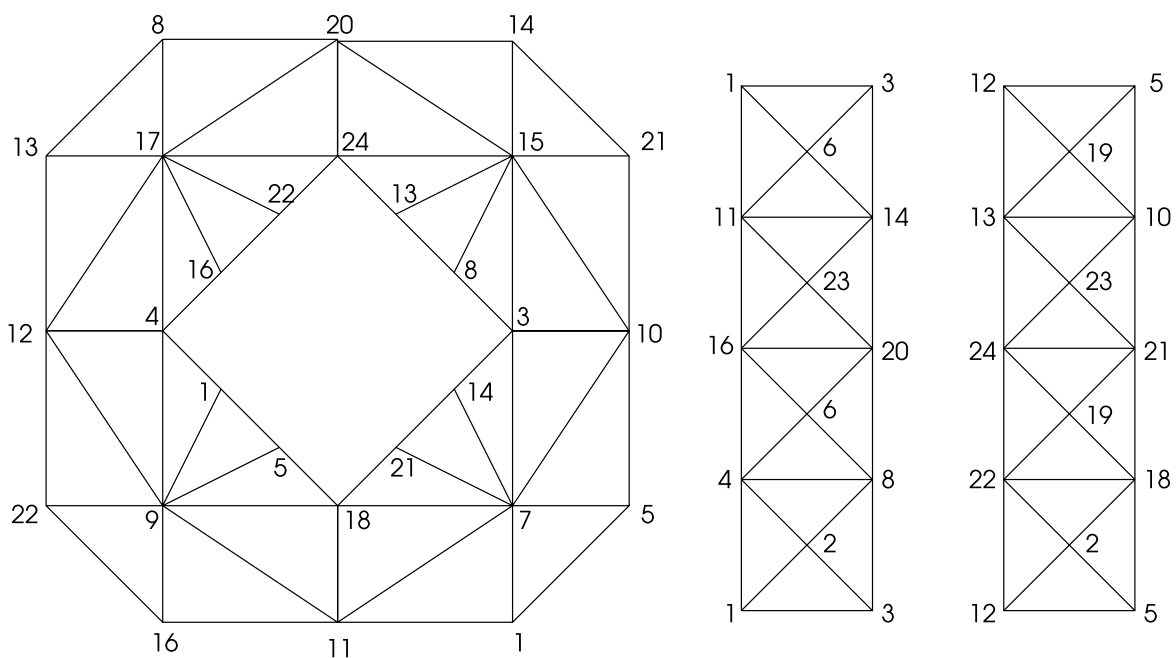

Fig. 2 The triangulation of the Hamiltonian pinched surface of genus 3 in the 24-cell
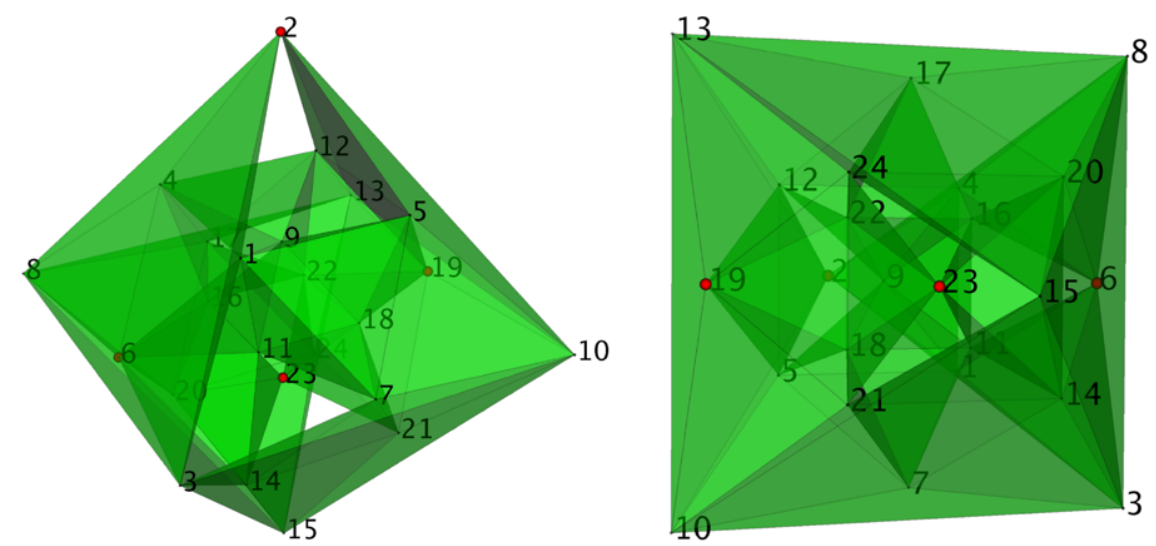

Fig. 3 Two projections of the Hamiltonian pinched surface of genus 3 in the 24-cell

(namely, two out of three). The same Euler characteristic would hold for a pinched surface if there is any. We remark that similarly the 4-cube admits a Hamiltonian surface of the same genus (namely, $g=1$ ) as the 4-dimensional cross polytope.

Proposition 2 There is no 1-Hamiltonian surface in the 2-skeleton of the 120-cell. There is no pinched surface either since the vertex link of the 120-cell is too small for containing two disjoint circuits. 
The proof is a fairly simple procedure: In each vertex link of type $\{3,3\}$ the Hamiltonian surface appears as a Hamiltonian circuit of length 4 . This is unique, up to symmetries of the tetrahedron and of the 120-cell itself. Note that two consecutive edges determine the circuit completely. So without loss of generality we can start with such a unique vertex link of the surface. This means that we start with four pentagons covering the star of one vertex. In each of the four neighboring vertices this determines two consecutive edges of the link there. It follows that these circuits are uniquely determined as well and that we can extend the beginning part of our surface, now covering the stars of five vertices. Successively this leads to a construction of such a surface. However, after a few steps it ends at a contradiction. Consequently, such a Hamiltonian surface does not exist.

Proposition 3 There is no 1-Hamiltonian surface in the 2-skeleton of the 600-cell.

This proof is more involved since it uses the classification of all 17 distinct Hamiltonian circuits in the icosahedron, up to symmetries of it [15, pp. $277 \mathrm{ff}$.]. If there is such a 1-Hamiltonian surface, then the link of each vertex in it must be a Hamiltonian cycle in the vertex link of the 600-cell which is an icosahedron. We just have to see how these can fit together. Starting with one arbitrary link, one can try to extend the triangulation to the neighbors. For the neighbors, there are forbidden 2-faces, which has a consequence for the possible types among the 17 for them. After an exhaustive computer search it turned out that there is no way to fit all vertex links together. Therefore such a surface does not exist. At this point it must be left open whether there are 1-Hamiltonian pinched surfaces in the 600-cell. The reason is that there are too many possibilities for a splitting into two, three, or four cycles in the vertex link. For a systematic search, one would have to classify all these possibilities first.

The GAP programs used for the algorithmic proof of Propositions 1, 2, 3 and details of the calculations are available from the first author upon request.

\section{Hamiltonian Submanifolds of Cross Polytopes}

The $d$-dimensional cross polytope $\beta^{d}$ (or the $d$-octahedron) is defined as the convex hull of the $2 d$ points

$$
(0, \ldots, 0, \pm 1,0, \ldots, 0) \in \mathbb{R}^{d} .
$$

It is a simplicial and regular polytope, and it is centrally-symmetric with $d$ diagonals, each between two antipodal points of type $(0, \ldots, 0,1,0, \ldots, 0)$ and $(0, \ldots, 0,-1,0, \ldots, 0)$. Its edge graph is the complete $d$-partite graph with two vertices in each partition, sometimes denoted by $K_{2} * \cdots * K_{2}$. See [31] for properties of regular polytopes in general. The $f$-vector of the cross polytope satisfies the equality

$$
f_{i}\left(\beta^{d}\right)=2^{i+1}\left(\begin{array}{c}
d \\
i+1
\end{array}\right) .
$$

Consequently, any 1-Hamiltonian 2-manifold must have the following beginning part of the $f$-vector:

$$
f_{0}=2 d, \quad f_{1}=2 d(d-1) .
$$


Table 3 Regular cases of 1-Hamiltonian 2-manifolds

\begin{tabular}{lrl}
\hline$d$ & $2-\chi$ & Genus $g$ \\
\hline 3 & 0 & 0 \\
4 & 2 & 1 \\
6 & 10 & 5 \\
7 & 16 & 8 \\
9 & 32 & 16 \\
10 & 42 & $3 \cdot 7=21$ \\
12 & 66 & $3 \cdot 11=33$ \\
13 & 80 & $8 \cdot 5=40$ \\
15 & 112 & $8 \cdot 7=56$ \\
16 & 120 & $4 \cdot 3 \cdot 5=60$ \\
18 & 170 & $5 \cdot 17=85$ \\
19 & 192 & $32 \cdot 3=96$ \\
21 & 240 & $8 \cdot 3 \cdot 5=120$ \\
22 & 266 & $7 \cdot 19=133$ \\
\hline
\end{tabular}

It follows that the Euler characteristic $\chi$ of the 2-manifold satisfies

$$
2-\chi=2-2 d+2 d(d-1)-\frac{4}{3} d(d-1)=\frac{2}{3}(d-1)(d-3) .
$$

These are the regular cases investigated in [17]. In terms of the genus $g=\frac{1}{2}(2-\chi)$ of an orientable surface, this equation reads as

$$
g=\frac{d-1}{1} \cdot \frac{d-3}{3}
$$

This remains valid for nonorientable surfaces if we assign the genus $\frac{1}{2}$ to the real projective plane. In any case, $\chi$ can be an integer only if $d \equiv 0,1$ (3). The first possibilities, where all cases are actually realized by triangulations of closed orientable surfaces [17], are indicated in Table 3.

Similarly, any 2-Hamiltonian 4-manifold must have the following beginning part of the $f$-vector:

$$
f_{0}=2 d, \quad f_{1}=2 d(d-1), \quad f_{2}=\frac{4}{3} d(d-1)(d-2) .
$$

It follows that the Euler characteristic $\chi$ satisfies

$$
\begin{aligned}
10(\chi-2) & =f_{2}-4 f_{1}+10 f_{0}-20=\frac{4}{3} d(d-1)(d-2)-8 d(d-1)+20 d-20 \\
& =\frac{4}{3}(d-1)(d-3)(d-5) .
\end{aligned}
$$

If we introduce the "genus" $g=\frac{1}{2}(\chi-2)$ of a simply connected 4-manifold as the number of copies of $S^{2} \times S^{2}$ which are necessary to form a connected sum with Euler 
characteristic $\chi$, then this equation reads as

$$
g=\frac{d-1}{1} \cdot \frac{d-3}{3} \cdot \frac{d-5}{5} .
$$

These are the "regular cases." Again the complex projective plane would have genus $\frac{1}{2}$ here. Recall that any 2-Hamiltonian 4-manifold in the boundary of a convex polytope is simply connected since the 2-skeleton is. Therefore the "genus" equals half of the second Betti number.

Moreover, there is an Upper Bound Theorem and a Lower Bound Theorem as follows:

Theorem 1 (Sparla [41]) If a triangulation of a 4-manifold occurs as a 2Hamiltonian subcomplex of a centrally-symmetric simplicial d-polytope, then the following inequality holds:

$$
\frac{1}{2}(\chi(M)-2) \geq \frac{d-1}{1} \cdot \frac{d-3}{3} \cdot \frac{d-5}{5} .
$$

Moreover, for $d \geq 6$, the equality is possible only if the polytope is affinely equivalent to the d-dimensional cross polytope.

If there is a triangulation of a 4-manifold with a fixed-point-free involution, then the number $n$ of vertices is even, i.e., $n=2 d$, and the opposite inequality holds:

$$
\frac{1}{2}(\chi(M)-2) \leq \frac{d-1}{1} \cdot \frac{d-3}{3} \cdot \frac{d-5}{5} .
$$

Moreover, the equality in this inequality implies that the manifold can be regarded as a 2-Hamiltonian subcomplex of the d-dimensional cross polytope.

Remark The case of equality in either of these inequalities corresponds to the "regular cases." Sparla's original equation $4^{3}\left(\begin{array}{c}\frac{1}{2}(d-1) \\ 3\end{array}\right)=10(\chi(M)-2)$ is equivalent to the one above.

By analogy, any $k$-Hamiltonian $2 k$-manifold in the $d$-dimensional cross polytope satisfies the equation

$$
(-1)^{k} \frac{1}{2}(\chi-2)=\frac{d-1}{1} \cdot \frac{d-3}{3} \cdot \frac{d-5}{5} \cdots \frac{d-2 k-1}{2 k+1} .
$$

It is necessarily $(k-1)$-connected, which implies that the left-hand side is half of the middle Betti number which is nothing but the "genus." Furthermore, there is a conjectured Upper Bound Theorem and a Lower Bound Theorem generalizing Theorem 1 where the inequality has to be replaced by

$$
(-1)^{k} \frac{1}{2}(\chi-2) \geq \frac{d-1}{1} \cdot \frac{d-3}{3} \cdot \frac{d-5}{5} \cdots \frac{d-2 k-1}{2 k+1}
$$

or

$$
(-1)^{k} \frac{1}{2}(\chi-2) \leq \frac{d-1}{1} \cdot \frac{d-3}{3} \cdot \frac{d-5}{5} \cdots \frac{d-2 k-1}{2 k+1},
$$


Table 4 Regular cases of 2-Hamiltonian 4-manifolds

\begin{tabular}{rrll}
\hline$d$ & $\chi-2$ & Genus $g$ & Existence \\
\hline 5 & 0 & 0 & $S^{4}=\partial \beta^{5}$ \\
6 & 2 & 1 & $S^{2} \times S^{2}[29,41]$ \\
8 & 14 & 7 & new (Theorem 2) \\
10 & 42 & $3 \cdot 7=21$ & see Remark 2 \\
11 & 64 & 32 & $?$ \\
13 & 128 & 64 & $?$ \\
15 & 224 & $16 \cdot 7=112$ & $?$ \\
16 & 286 & $11 \cdot 13=143$ & $?$ \\
18 & 442 & $13 \cdot 17=221$ & $?$ \\
20 & 646 & $17 \cdot 19=323$ & $?$ \\
21 & 720 & $8 \cdot 5 \cdot 9=360$ & $?$ \\
23 & 1056 & $16 \cdot 3 \cdot 11=528$ & $?$ \\
25 & 1408 & $64 \cdot 11=704$ & $?$ \\
26 & 1610 & $5 \cdot 7 \cdot 23=805$ & $?$ \\
28 & 2070 & $5 \cdot 9 \cdot 23=1035$ & $?$ \\
30 & 2610 & $5 \cdot 9 \cdot 29=1305$ & $?$ \\
\hline
\end{tabular}

respectively, see $[33,42]$. The discussion of the cases of equality is exactly the same. Sparla's original version

$$
4^{k+1}\left(\begin{array}{c}
\frac{1}{2}(d-1) \\
k+1
\end{array}\right)=\left(\begin{array}{c}
2 k+1 \\
k+1
\end{array}\right)(-1)^{k}(\chi(M)-2)
$$

is equivalent to the one above. In particular, for any $k$, one of the "regular cases" is the case of a sphere product $S^{k} \times S^{k}$ with $(-1)^{k}(\chi-2)=2$ (or "genus" $g=1$ ) and $d=2 k+2$. So far examples are available for $1 \leq k \leq 4$, even with a vertex transitive automorphism group see [27, 30]. We hope that for $k \geq 5$, there will be similar examples as well, compare Sect. 6.

\section{2-Hamiltonian 4-Manifolds in Cross Polytopes}

In the case of 2-Hamiltonian 4-manifolds as subcomplexes of the $d$-dimensional cross polytope, we have the "regular cases" of the equality $g=\frac{1}{2}(\chi-2)=\frac{d-1}{1} \cdot \frac{d-3}{3} \cdot \frac{d-5}{5}$. Here $\chi$ can be an integer only if $d \equiv 0,1,3$ (5). Table 4 indicates the first possibilities.

Theorem 2 There is a 16-vertex triangulation of a 4-manifold $M \cong\left(S^{2} \times S^{2}\right)^{\# 7}$ which can be regarded as a centrally-symmetric and 2-Hamiltonian subcomplex of the 8-dimensional cross polytope. As one of the "regular cases," it satisfies the equality in Sparla's inequalities in Theorem 1 with "genus" $g=7$ and with $d=8$. 
Proof Any 2-Hamiltonian subcomplex of a convex polytope is simply connected [20, 3.8]. Therefore such an $M$, if it exists, must be simply connected, in particular $H_{1}(M)=H_{3}(M)=0$. In accordance with Sparla's inequalities, the Euler characteristic $\chi(M)=16$ tells us that the middle homology group is $H_{2}(M, \mathbb{Z}) \cong \mathbb{Z}^{14}$. The topological type of $M$ is then uniquely determined by the intersection form. If the intersection form is even, then by Rohlin's theorem the signature must be zero, which implies that $M$ is homeomorphic to the connected sum of 7 copies of $S^{2} \times S^{2}$, see [37]. If the intersection form is odd, then $M$ is a connected sum of 14 copies of $\pm \mathbb{C} P^{2}$. We will show that the intersection form of our example is even.

The induced polyhedral embedding of this manifold into 8-space is tight since the intersection with any open halfspace is connected and simply connected, compare Sect. 5 below. No smooth tight embedding of this manifold into 8-space can exist, see [44]. Consequently, this embedding of $M$ into 8-space is smoothable as far as the PL structure is concerned, but it is not tightly smoothable.

The $f$-vector $f=(16,112,448,560,224)$ of this example is uniquely determined already by the requirement of 16 vertices and the condition to be 2-Hamiltonian in the 8 -dimensional cross polytope. In particular there are 8 missing edges corresponding to the 8 diagonals of the cross polytope which are pairwise disjoint.

Assuming a vertex-transitive automorphism group, an example was found by using the software of Lutz described in [30]. The combinatorial automorphism group $G$ of our example is of order 128. With this particular automorphism group the example is unique. The special element

$$
\zeta=(12)(34)(5 \text { 6)(7 8)(9 10)(11 12)(13 14)(15 16) }
$$

acts on $M$ without fixed points. It interchanges the endpoints of each diagonal and, therefore, can be regarded as the antipodal mapping sending each vertex of the 8dimensional cross polytope to its antipodal vertex in such a way that it is compatible with the subcomplex $M$. A normal subgroup $H$ isomorphic to $C_{2} \oplus C_{2} \oplus C_{2} \oplus C_{2}$ acts simply transitively on the 16 vertices. The isotropy group $G_{0}$ fixing one vertex (and, simultaneously, its antipodal vertex) is isomorphic to the dihedral group of order 8. The group itself is a semidirect product between $H$ and $G_{0}$. In more detail the example is given by the three $G$-orbits of the 4 -simplices

$$
\langle 13579\rangle_{128}, \quad\langle 135913\rangle_{64}, \quad\langle 135715\rangle_{32}
$$

with altogether $128+64+32=224$ simplices, each given by a 5 -tuple of vertices out of $\{1,2,3, \ldots, 15,16\}$. The group $G \cong\left(\left(\left(\left(C_{4} \oplus C_{2}\right): C_{2}\right): C_{2}\right): C_{2}\right): C_{2}$ of order 128 is generated by the three permutations $\alpha=\left(\begin{array}{lllll}1 & 12 & 16 & 14 & 2 \\ 11 & 15 & 13\end{array}\right)$ $\left(\begin{array}{llllllll}3 & 10 & 6 & 8 & 4 & 9 & 5 & 7\end{array}\right), \beta=\left(\begin{array}{llll}1 & 6 & 2 & 5\end{array}\right)\left(\begin{array}{llll}7 & 9 & 2 & 14\end{array}\right)\left(\begin{array}{llll}8 & 10 & 11 & 13\end{array}\right)\left(\begin{array}{lll}15 & 16\end{array}\right), \gamma=$ (1 123 14)(2 11413$)(571610)(68159)$. 
The complete list of all 224 top-dimensional simplices is the following:

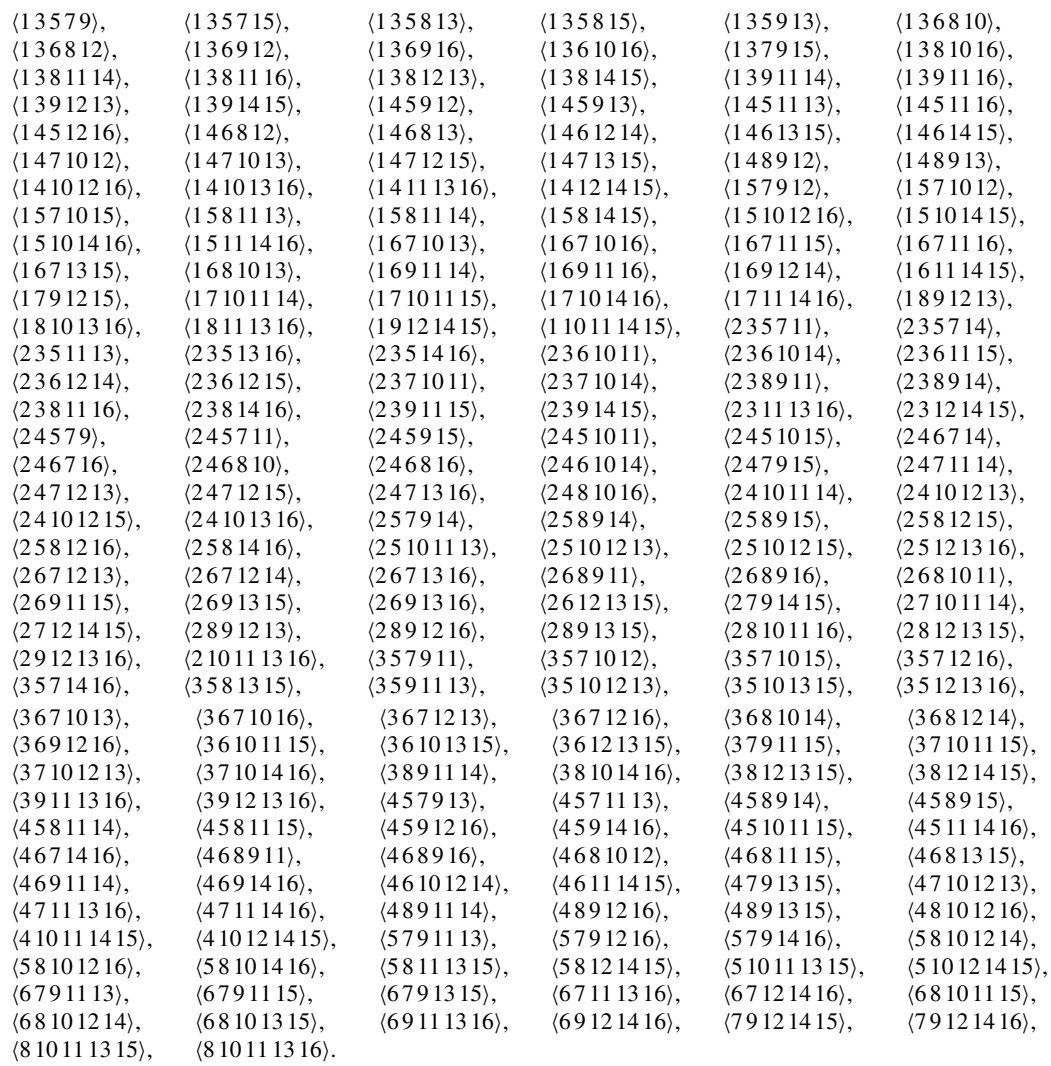

The link of the vertex 16 is the following simplicial 3-sphere with 70 tetrahedra:

$\begin{array}{llllllll}\langle 1369\rangle, & \langle 13610\rangle, & \langle 13810\rangle, & \langle 13811\rangle, & \langle 13911\rangle, & \langle 14511\rangle, & \langle 14512\rangle, & \langle 141012\rangle, \\ \langle 141013\rangle, & \langle 141113\rangle, & \langle 151012\rangle, & \langle 151014\rangle, & \langle 151114\rangle, & \langle 16710\rangle, & \langle 16711\rangle, & \langle 16911\rangle, \\ \langle 171014\rangle, & \langle 171114, & \langle 181013\rangle, & \langle 181113\rangle, & \langle 23513\rangle, & \langle 23514, & \langle 23811\rangle, & \langle 23814\rangle, \\ \langle 231113\rangle, & \langle 2467\rangle, & \langle 2468\rangle, & \langle 24713\rangle, & \langle 24810\rangle, & \langle 241013\rangle, & \langle 25812\rangle, & \langle 25814\rangle, \\ \langle 251213\rangle, & \langle 26713\rangle, & \langle 2689\rangle, & \langle 26913\rangle, & \langle 28912\rangle, & \langle 281011\rangle, & \langle 291213\rangle, & \langle 2101113\rangle, \\ \langle 35712\rangle, & \langle 35714\rangle, & \langle 351213\rangle, & \langle 36710\rangle, & \langle 36712\rangle, & \langle 36912\rangle, & \langle 371014\rangle, & \langle 381014\rangle, \\ \langle 391113\rangle, & \langle 391213\rangle, & \langle 45912\rangle, & \langle 45914\rangle, & \langle 451114\rangle, & \langle 46714\rangle, & \langle 4689\rangle, & \langle 46914\rangle, \\ \langle 471113\rangle, & \langle 471114\rangle, & \langle 48912\rangle, & \langle 481012\rangle, & \langle 57912\rangle, & \langle 57914\rangle, & \langle 581012\rangle, & \langle 581014\rangle, \\ \langle 671113\rangle, & \langle 671214\rangle, & \langle 691113\rangle, & \langle 691214\rangle, & \langle 791214\rangle, & \langle 8101113\rangle, & & \end{array}$

It remains to prove two facts:

Claim 1 The link of the vertex 16 is a combinatorial 3-sphere. This implies that $M$ is a PL-manifold since all vertices are equivalent under the action of the automorphism group.

A computer algorithm gave a positive answer: the link of the vertex 16 is combinatorially equivalent to the boundary of a 4-simplex by bistellar moves. This method is described in [6] and [30, 1.3]. 
Claim 2 The intersection form of $M$ is even or, equivalently, the second StiefelWhitney class of $M$ vanishes. This implies that $M$ is homeomorphic to the connected sum of 7 copies of $S^{2} \times S^{2}$.

There is an algorithm for calculating the second Stiefel-Whitney class [14]. There is also an computer algorithm implemented in polymake [13], compare [16] for determining the intersection form itself. The latter algorithm gave the following answer: The intersection form of $M$ is even, and the signature is zero.

In order to illustrate the intersection form on the second homology, we consider the link of the vertex 16, as given above. By the tightness condition special homology classes are represented by the empty tetrahedra $c_{1}=\langle 7101116\rangle$ and $d_{1}=\langle 8121316\rangle$, which are interchanged by the element

$$
\delta=\left(\begin{array}{ll}
1 & 2
\end{array}\right)\left(\begin{array}{lll}
5 & 6
\end{array}\right)(7 \text { 12)(8 11)(9 14)(10 13) }
$$

of the automorphism group. The intersection number of these two equals the linking number of the empty triangles $\langle 71011\rangle$ and $\langle 81213\rangle$ in the link of 16 . The two subsets in the link spanned by $1,5,7,10,11,14$ and $2,6,8,9,12,13$, respectively, are homotopy circles interchanged by $\delta$. The intermediate subset of points in the link of 16 which is invariant under $\delta$ is the torus depicted in Fig. 4. The set of points which are fixed by $\delta$ are represented as the horizontal $(1,1)$-curve in this torus, the element $\delta$ itself appears as the reflection along that fixed curve. This torus shrinks down to the homotopy circle on either of the sides which are spanned by $1,5,7,10,11,14$ and $2,6,8,9,12,13$, respectively. The empty triangles $\langle 71011\rangle$ and $\langle 81213\rangle$ also represent the same homotopy circles. Since the link is a 3-sphere, these two are linked with linking number \pm 1 . As a result, we get for the intersection form $c_{1} \cdot d_{1}= \pm 1$. These two empty tetrahedra $c_{1}$ and $d_{1}$ are not homologous to one another in $M$. Each one can be perturbed into a disjoint position such that the self linking number is zero: $c_{1} \cdot c_{1}=d_{1} \cdot d_{1}=0$. Therefore $c_{1}, d_{1}$ represent a part of the intersection form isomorphic with $\pm\left(\begin{array}{ll}0 & 1 \\ 1 & 0\end{array}\right)$. This situation is transferred to the intersection form of other generators by the automorphism group. As a result, we have seven copies of the matrix as a direct sum.

Remark 1 Looking at the action of the automorphism group $G$ on the free abelian group $H_{2}(M, \mathbb{Z}) \cong \mathbb{Z}^{14}$, we get on the 17 conjugacy classes of $G$ the following character values:

$$
(14,-2,-2,-2,2,-2,6,-2,-2,-2,6,0,0,0,0,0,0) \text {. }
$$

Denote by $\chi$ the corresponding ordinary character. Using the character table ${ }^{1}$ of $G$ given by GAP [43] and the orthogonality relations, this character decomposes into a sum of five irreducible ordinary characters as follows:

$$
\chi=\chi_{2}+\chi_{3}+\chi_{13}+\chi_{14}+\chi_{17}
$$

\footnotetext{
${ }^{1}$ We thank Wolfgang Kimmerle for helpful comments concerning group representations.
} 


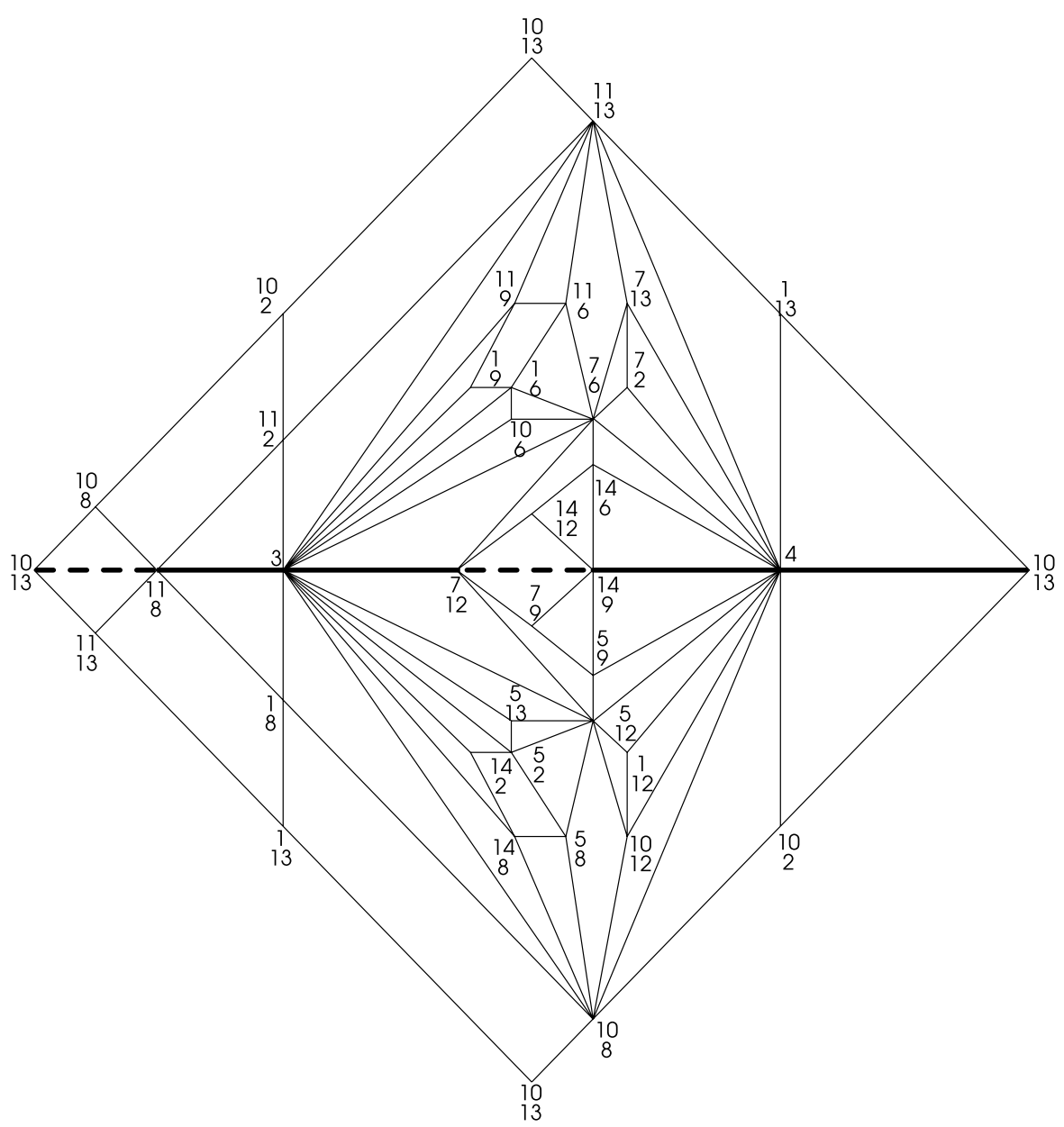

Fig. 4 The intermediate torus in the link of 16, invariant under the reflection $\delta$

This shows that $\mathbb{C} \bigotimes_{\mathbb{Z}} H_{2}(M, \mathbb{Z})$ is a cyclic $\mathbb{C} G$-module. It may be interesting to find a geometric explanation for this. The involved irreducible characters are as follows:

\begin{tabular}{l|rrrrrrrrrrrrrrrrr} 
& $1 a$ & $2 a$ & $2 b$ & $2 c$ & $4 a$ & $2 d$ & $2 e$ & $4 b$ & $4 c$ & $4 d$ & $2 f$ & $4 e$ & $4 f$ & $4 g$ & $4 h$ & $8 a$ & $2 g$ \\
\hline$\chi_{2}$ & 1 & -1 & 1 & -1 & 1 & 1 & 1 & -1 & 1 & -1 & 1 & -1 & 1 & -1 & 1 & -1 & 1 \\
$\chi_{3}$ & 1 & -1 & 1 & -1 & 1 & 1 & 1 & -1 & 1 & -1 & 1 & 1 & -1 & 1 & -1 & 1 & -1 \\
$\chi_{13}$ & 2 &. & -2 &. &. & 2 & 2 &. & -2 &. & 2 & -2 &. & 2 &. &. &. \\
$\chi_{14}$ & 2 &. & -2 &. &. & 2 & 2 &. & -2 &. & 2 & 2 &. & -2 &. &. &. \\
$\chi_{17}$ & 8 &. &. &. &. & -8 &. &. &. &. &. &. &. &. &. &. &.
\end{tabular}

Remark 2 There is a real chance to solve the next regular case $d=10$ in Sparla's inequality. The question is whether there is a 2-Hamiltonian 4-manifold of genus 21 (i.e., $\chi=44$ ) in the 10 -dimensional cross polytope. A 22-vertex triangulation of a 
manifold with exactly the same genus as a subcomplex of the 11-dimensional cross polytope does exist. If one could save two antipodal vertices by successive bistellar flips, one would have a solution. The example with 22 vertices is defined by the orbits (of length 110 or 22, respectively) of the 4-simplices

$$
\begin{array}{lll}
\langle 135718\rangle_{110}, & \langle 135721\rangle_{110}, & \langle 135818\rangle_{110}, \\
\langle 135821\rangle_{110}, & \langle 1371820\rangle_{110}, & \langle 1361015\rangle_{22}
\end{array}
$$

under the permutation group of order 110 which is generated by

(11672213519123181021582114620114179)

and

$$
\text { (1 } 1117321)(21218422)(5982014)(61071913) \text {. }
$$

The central involution is

$$
(12)(34)(5 \text { 6)(7 8)(9 10)(11 12)(13 14)(15 16)(17 18)(19 20)(21 22), }
$$

which corresponds to the antipodal mapping in the cross polytope. The $f$-vector of the example is $(22,220,1100,1430,572)$, and the middle homology is 42 dimensional, the first and third homologies both vanish. Hence it has "genus" 21 in the sense defined above.

\section{Tightness and Tautness}

The concept of tightness originates from differential geometry as the equality of the (normalized) total absolute curvature of a submanifold with the lower bound sum of the Betti numbers [3, 28]. It is also a generalization of the concept of convexity since it roughly means that an embedding of a submanifold is as convex as possible according to its topology. The usual definition is the following:

Definition (Compare [28]) An embedding $M \rightarrow \mathbb{E}^{N}$ of a compact manifold is called tight if for any open or closed halfspace $E_{+}^{N} \subset \mathbb{E}^{N}$, the induced homomorphism

$$
H_{*}\left(M \cap E_{+}^{N}\right) \longrightarrow H_{*}(M)
$$

is injective, where $H_{*}$ denotes an appropriate homology theory with coefficients in a certain field. The notion of $k$-tightness refers to the injectivity in the low dimensions $H_{i}\left(M \cap E_{+}^{N}\right) \rightarrow H_{i}(M), i=0, \ldots, k$, see [20]. An equivalent formulation is that all nondegenerate height functions are perfect functions, i.e., functions with a number of critical points which equals the sum of the Betti numbers. This definition applies to smooth and polyhedral embeddings. A tight triangulation is a triangulation of a manifold such that any simplexwise linear embedding is tight [20, 27]. Any $k$-Hamiltonian $2 k$-manifold in the $d$-dimensional simplex is induced by a tight triangulation with $d+1$ vertices. For a subcomplex of the boundary complex of a convex polytope, the tightness condition is often determined by purely combinatorial conditions. In 
particular any $k$-Hamiltonian $2 k$-manifold in a $d$-polytope is tightly embedded into $d$-space [20, 4.1]. For any tight subcomplex $K$ of the boundary complex of a convex polytope, the following is a direct consequence of the definition above, compare $[20,1.4]$ :

Consequence 1 A facet of the polytope is either contained in $K$, or its intersection with $K$ represents a subset of $K$ (often called a topset) which injects into $K$ at the homology level and which is again tightly embedded into the ambient space. In particular, any missing $(k+1)$-simplex in a $k$-Hamiltonian subcomplex $K$ of a simplicial polytope represents a nonvanishing element of the kth homology by the standard triangulation of the $k$-sphere.

For the similar notion of tautness, one has to replace halfspaces by balls (or ball complements) $B$ and height functions by distance functions, see [10]. This applies only to smooth embeddings. In the polyhedral case it has to be modified as follows:

Definition (Suggested in [4]) A PL-embedding $M \rightarrow \mathbb{E}^{N}$ of a compact manifold with convex faces is called PL-taut if for any open ball (or ball complement) $B \subset \mathbb{E}^{N}$, the induced homomorphism

$$
H_{*}\left(M \cap \operatorname{span}\left(B_{0}\right)\right) \longrightarrow H_{*}(M)
$$

is injective, where $B_{0}$ denotes the set of vertices in $M \cap B$, and $\operatorname{span}\left(B_{0}\right)$ refers to the subcomplex in $M$ spanned by those vertices.

Obviously, any PL-taut embedding is also tight (consider very large balls), and a tight PL-embedding is PL-taut, provided that it is PL-spherical in the sense that all vertices are contained in a certain Euclidean sphere. It follows that any tight and PL-spherical embedding is also PL-taut [4].

Corollary Any tight subcomplex of a higher-dimensional regular simplex, cube, or cross polytope is PL-taut.

In particular this implies that the class of PL-taut submanifolds is much richer than the class of smooth taut submanifolds.

Corollary There is a tight and PL-taut simplicial embedding of the connected sum of 7 copies of $S^{2} \times S^{2}$ into Euclidean 8-space.

This follows directly from Theorem 2 by the embedding into the 8-dimensional cross polytope. In addition this example is centrally-symmetric. There is a standard construction of tight embeddings of connected sums of copies of $S^{2} \times S^{2}$, but this works in codimension 2 only, polyhedrally and smoothly, see [3, p. 101]. The cubical examples in [25] exist in arbitrary codimension, but they require a much larger "genus": For a 2-Hamiltonian 4-manifold in the 8-dimensional cube, one needs the Euler characteristic $\chi=64$, which corresponds to a connected sum of 31 copies of $S^{2} \times S^{2}$. The number of summands in this case grows exponentially with the dimension of the cube. For a 2-Hamiltonian 4-manifold in the 8-dimensional simplex, the 
Euler characteristic $\chi=3$ is sufficient. It is realized by the 9 -vertex triangulation of $\mathbb{C} P^{2}[23,24]$. One copy of $S^{2} \times S^{2}$ cannot be a subcomplex of the 9-dimensional simplex because such a 3-neighborly 10-vertex triangulation does not exist [24] even though it is one of the "regular cases" in the sense of the Heawood-type integer condition in Sect. 1. In general the idea behind is the following: A given $d$-dimensional polytope requires a certain minimum "genus" of a $2 k$-manifold to cover the full $k$ dimensional skeleton of the polytope. For the standard polytopes like simplex, $d$ cube, and $d$-octahedron, we have formulas for the "genus" which is to be expected, but we do not yet have examples in all of the cases.

The situation is similar with respect to the concept of tightness: For any given dimension $d$ of an ambient space, a certain "genus" of a manifold is required for admitting a tight and substantial embedding into $d$-dimensional space. This is well understood in the case of 2-dimensional surfaces [20]. For "most" of the simply connected 4-manifolds, a tight polyhedral embedding was constructed in [22], without any especially intended restriction concerning the essential codimension. The optimal bounds in this case and in all the other higher-dimensional cases still have to be investigated.

\section{Centrally-Symmetric Triangulations of Sphere Products}

As far as the integer conditions of the "regular cases" are concerned, it seems to be plausible to ask for centrally-symmetric triangulations of any sphere product $S^{k} \times S^{l}$ with a minimum number of

$$
n=2(k+l+2)
$$

vertices. In this case each instance can be regarded as a codimension-1-subcomplex of the boundary complex of the $(k+l+2)$-dimensional cross polytope, and it can be expected to be $m$-Hamiltonian for $m=\min (k, l)$. This is a kind of a simplicial Hopf decomposition of the $(k+l+1)$-sphere by "Clifford-tori" of type $S^{k} \times S^{l}$.

For $n \leq 20$ (i.e., for $k+l \leq 8$ ), a census of such triangulations with a vertextransitive automorphism group can be found in [30], compare [27]. Here all cases occur except for $S^{4} \times S^{2}$ and $S^{6} \times S^{2}$, and all examples admit a dihedral group action of order $2 n$. So far an infinite series of examples seems to be known only for $l=1$ and arbitrary $k$. This is the following:

Proposition 4 (A centrally-symmetric and 1-Hamiltonian $S^{k} \times S^{1}$ in $\partial \beta_{k+3}$ ) There is a centrally-symmetric triangulation of $S^{k} \times S^{1}$ with $n=2 k+6$ vertices and with a dihedral automorphism group $D_{n}$. Its induced embedding into the $(k+3)$-dimensional cross polytope is tight and PL-taut.

The construction is given in [26] with the notation $M_{k}^{k+1}(n)$ (represented as the permcycle [ $1^{k} 2$ ] there) as follows: Regard the vertices as integers modulo $n$ and consider the $\mathbb{Z}_{n}$-orbit of the $(k+2)$-simplex

$$
\langle 012 \cdots k(k+1)(k+2)\rangle \text {. }
$$

This is a manifold with boundary (just an ordinary orientable 1-handle), and its boundary is homeomorphic to $S^{k} \times S^{1}$. All these simplices are facets of the cross 
polytope of dimension $k+3$ if we choose the labeling such that the diagonals are $[x, x+k+3], x \in \mathbb{Z}_{n}$. These diagonals do not occur in the triangulation of the manifold, and all other edges are contained. Therefore we obtain a 1-Hamiltonian subcomplex of the $(k+3)$-dimensional cross polytope. The central symmetry is the shift $x \mapsto x+k+3$ in $\mathbb{Z}_{n}$. The reflection $x \mapsto-x$ in $\mathbb{Z}_{n}$ is an extra automorphism. In the case $k=1$ the group is even larger: It is of order 32. This triangulated manifold is a hypersurface in $\partial \beta_{k+3}$, it decomposes this $(k+2)$-sphere into two parts with the same topology as suggested by the Hopf decomposition.

The same generating simplex for the group $\mathbb{Z}_{m}$ with $m=2 k+5$ vertices leads to the minimum vertex triangulation of $S^{k} \times S^{1}$ (for odd $k$ ) or of the twisted product (for even $k$ ), which is actually unique $[2,11]$. For any $k \geq 2$, it realizes the minimum number of vertices for any manifold of the same dimension which is not simply connected [7]. Other infinite series of triangulated sphere bundles over tori are similarly given in [26].

It is not impossible that there will be direct generalizations of Proposition 4 with infinite series of analogous triangulations of $S^{k} \times S^{3}, S^{k} \times S^{5}, \ldots$, at least for odd $k$, and of $S^{k} \times S^{k}$, possibly for any $k$, each with a dihedral and vertex-transitive group action and Hamiltonian in the cross polytope. This is still work in progress. The existence in the latter case of a $k$-Hamiltonian $S^{k} \times S^{k}$ with $n=4 k+4$ vertices and $d=2 k+2$ would give a positive answer to a conjecture by Lutz [30, p. 85] and would realize the equality in Sparla's inequality in Sect. 3 for any $k$ since

$$
(-1)^{k} \frac{1}{2}(\chi-2)=1=\frac{2 k+1}{1} \cdot \frac{2 k-1}{3} \cdot \frac{2 k-3}{5} \cdots \frac{1}{2 k+1} .
$$

Acknowledgements We acknowledge support by the Deutsche Forschungsgemeinschaft (DFG). This work was carried out as part of the DFG-project Ku 1203/5.

\section{References}

1. Altshuler, A.: Manifolds in stacked 4-polytopes. J. Comb. Theory A 10, 198-239 (1971)

2. Bagchi, B., Datta, B.: Minimal triangulations of sphere bundles over the circle. J. Comb. Theory A 115, 737-752 (2008)

3. Banchoff, T.F., Kühnel, W.: Tight submanifolds, smooth and polyhedral. In: Cecil, T.E., Chern, S.-S. (eds.) Tight and Taut Submanifolds. MSRI Publ., vol. 32, pp. 51-118. Cambridge University Press, Cambridge (1997)

4. Banchoff, T.F., Kühnel, W.: Tight polyhedral models of isoparametric families, and PL-taut embeddings. Adv. Geom. 7, 613-629 (2007)

5. Beineke, L.W., Harary, F.: The genus of the $n$-cube. Can. J. Math. 17, 494-496 (1965)

6. Björner, A., Lutz, F.H.: Simplicial manifolds, bistellar flips and a 16-vertex triangulation of the Poincaré homology 3-sphere. Exp. Math. 9, 275-289 (2000)

7. Brehm, U., Kühnel, W.: Combinatorial manifolds with few vertices. Topology 26, 465-473 (1987)

8. Brehm, U., Kühnel, W.: 15-vertex triangulations of an 8-manifold. Math. Ann. 294, 167-193 (1992)

9. Casella, M., Kühnel, W.: A triangulated $K 3$ surface with the minimum number of vertices. Topology 40, 753-772 (2001)

10. Cecil, T.E., Ryan, P.J.: Tight and Taut Immersions of Manifolds. Pitman, London (1985)

11. Chestnut, J., Sapir, J., Swartz, E.: Enumerative properties of triangulations of sphere bundles over $S^{1}$. Eur. J. Comb. 29(1), 662-667 (2008)

12. Ewald, G.: Hamiltonian circuits in simplicial complexes. Geom. Dedic. 2, 115-125 (1973)

13. Gawrilow, E., Joswig, M.: Polymake: a framework for analyzing convex polytopes. In: PolytopesCombinatorics and Computation (Oberwolfach, 1997). DMV Sem., vol. 29, pp. 43-73. Birkhäuser, Basel (2000) 
14. Goldstein, R.Z., Turner, E.C.: A formula for Stiefel-Whitney homology classes. Proc. Am. Math. Soc. 58, 339-342 (1976)

15. Heesch, H.: Gesammelte Abhandlungen. Franzbecker, Hildesheim (1986)

16. Joswig, M.: Computing invariants of simplicial manifolds. Preprint (2004). arXiv:math/0401176 [math.AT]

17. Jungerman, M., Ringel, G.: The genus of the $n$-octahedron: Regular cases. J. Graph Theory 2, 69-75 (1978)

18. Koh, K.M., Teo, K.L.: The 2-Hamiltonian cubes of graphs. J. Graph Theory 13, 737-747 (1989)

19. Kühnel, W.: Manifolds in the skeletons of convex polytopes, tightness, and generalized Heawood inequalities. In: Bisztriczky, T. et al. (eds.) POLYTOPES: Abstract, Convex and Computational, Proc. Conf. Scarborough 1993. NATO Adv. Study Inst. Ser. C, Math. Phys. Sci., vol. 440, pp. 241-247. Kluwer Academic, Dordrecht (1994)

20. Kühnel, W.: Tight Polyhedral Submanifolds and Tight Triangulations. Lecture Notes in Mathematics, vol. 1612. Springer, Berlin (1995)

21. Kühnel, W.: Centrally-symmetric tight surfaces and graph embeddings. Beiträge Algebra Geom. 37, 347-354 (1996)

22. Kühnel, W.: Tight embeddings of simply connected 4-manifolds. Doc. Math. 9, 401-412 (2004)

23. Kühnel, W., Banchoff, T.F.: The 9-vertex complex projective plane. Math. Intell. 5(3), 11-22 (1983)

24. Kühnel, W., Lassmann, G.: The unique 3-neighborly 4-manifold with few vertices. J. Comb. Theory A 35, 173-184 (1983)

25. Kühnel, W., Schulz, C.: Submanifolds of the cube. In: Gritzmann, P., Sturmfels, B. (eds.) Applied Geometry and Discrete Mathematics, The Victor Klee Festschrift. DIMACS Series in Discrete Math. and Theor. Comp. Sci., vol. 4, pp. 423-432. Am. Math. Soc., Providence (1991)

26. Kühnel, W., Lassmann, G.: Permuted difference cycles and triangulated sphere bundles. Discrete Math. 162, 215-227 (1996)

27. Kühnel, W., Lutz, F.H.: A census of tight triangulations. Period. Math. Hung. 39, 161-183 (1999)

28. Kuiper, N.H.: Geometry in total absolute curvature theory. In: Jäger, M. et al. (eds.) Perspectives in Mathematics, Anniversary of Oberwolfach 1984, pp. 377-392. Birkhäuser, Basel (1984)

29. Lassmann, G., Sparla, E.: A classification of centrally-symmetric and cyclic 12-vertex triangulations of $S^{2} \times S^{2}$. Discrete Math. 223, 175-187 (2000)

30. Lutz, F.H.: Triangulated manifolds with few vertices and vertex-transitive group actions. Doctoral Thesis TU Berlin 1999. Shaker, Aachen (1999)

31. McMullen, P., Schulte, E.: Abstract Regular Polytopes. Cambridge University Press, Cambridge (2002)

32. Novik, I.: Upper bound theorems for homology manifolds. Isr. J. Math. 108, 45-82 (1998)

33. Novik, I.: On face numbers of manifolds with symmetry. Adv. Math. 192, 183-208 (2005)

34. Novik, I., Swartz, E.: Socles of Buchsbaum modules, complexes and posets. Preprint (2007). arXiv:0711.0783 [math.CO]

35. Ringel, G.: Über drei Probleme am $n$-dimensionalen Würfel und Würfelgitter. Abh. Math. Sem. Univ. Hamb. 20, 10-19 (1955)

36. Ringel, G.: Map Color Theorem. Springer, Berlin (1974)

37. Saveliev, N.: Lectures on the Topology of 3-Manifolds. An Introduction to the Casson Invariant. de Gruyter, Berlin (1999)

38. Schulz, C.: Mannigfaltigkeiten mit Zellzerlegung im Randkomplex eines konvexen Polytops und verallgemeinerte Hamilton-Kreise. Doctoral Dissertation Univ. Bochum (1974)

39. Schulz, C.: Polyhedral manifolds on polytopes, Proc. Conf. Palermo 1993. Rend. Circ. Mat. Palermo (2) Suppl. 35, 291-298 (1993)

40. Séquin, C.H.: Symmetrical Hamiltonian manifolds on regular 3D and 4D polytopes. The Coxeter Day, Banff, Canada, 3 Aug. 2005, pp. 463-472 (2005). See http://www.cs.berkeley.edu/ sequin/ $\mathrm{BIO} /$ pubs.html

41. Sparla, E.: An upper and a lower bound theorem for combinatorial 4-manifolds. Discrete Comput. Geom. 19, 575-593 (1998)

42. Sparla, E.: A new lower bound theorem for combinatorial $2 k$-manifolds. Graphs Comb. 15, 109-125 (1999)

43. The GAP Group: GAP-Groups, Algorithms and Programming, Version 4.2. Aachen, St. Andrews (1999). See http://www.gap-system.org

44. Thorbergsson, G.: Tight immersions of highly connected manifolds. Comment. Math. Helv. 61, 102121 (1986) 